Original Research Paper

\title{
A Novel Approach to Wheelchair Design and Operation Using Multi-Function Controller
}

\author{
Jamal I. Al-Nabulsi \\ Department of Medical Engineering, Al-Ahliyya Amman University, Amman, Jordan
}

\author{
Article history \\ Received: 05-05-2020 \\ Revised: 04-07-2020 \\ Accepted: 23-07-2020 \\ Email: j.nabulsi@ammanu.edu.jo
}

\begin{abstract}
An electric multi-function controlled wheelchair is designed and fully tested. This wheelchair is controlled by several physiological variables namely; voice, head movement, finger bending, breathing pressure and Electrooculography (EOG). The patient has the choice to use any of these variables to control the wheelchair. The voice command is recorded by a voice recognition module with its microphone, whereas, the head and finger motion operate through the gyro accelerometer and flex sensors. A pressure sensor is used to determine the force of breathing and EOG signals are used to control the wheelchair movement. All of the inputs are processed using a microcontroller. Testing of the wheel chair using the mentioned variables is carried out successfully with accuracy between 88 to $96 \%$ for various control modules and safety consideration as a primary goal.
\end{abstract}

Keywords: Wheelchair, Breathing-Pressure, EOG, Head-Motion, Voice Recognition Module, Body Gestures

\section{Introduction}

For the past few decades, the need for assistive devices has increased numerously because of the different injuries that could occur to people globally starting from work related injuries to war related injuries. The wheelchair is one of the most commonly used assistive devices for enhancing personal mobility. Wheelchairs assist people with disabilities to become productive members of their communities.

According to the US census, around $10 \%$ of the global population has disabilities and require some kind of assistive device (Taylor, 2018), where in Europe, according to several statistics is estimated to be around $1 \%$. These estimates reach between 5 to $10 \%$ in countries like Cambodia or Afghanistan (Philip, 2012). The current formal estimate of disability incidence in Jordan is more than $10 \%$ as reported in (Thompson, 2018).

The first commercially manufactured electric wheelchair was simply heavy-duty powered by leadacid batteries, engines, drive belts and pulleys (Mann et al., 2002). Advances in batteries and electric system management which covers engines and batteries enabled important mechanical advances in electric wheelchairs, where the power base divided the electric wheelchair into two components: The base providing the mobility and the seating system providing the support for the posture, while there was a change from a standard power wheelchair to a power-base wheelchair, (Ebrahimi et al., 2016) reported important developments in electronic devices. Chow and Levy (2011) have shown some of these mechanical and electrical advances included the capacity to add energy tilt and recline systems, as well as programmable performance settings such as forward speed, turning velocity and velocity.

New control techniques were developed that surpass using joystick to control a powered wheelchair; most common solution's is a control using the chin or elbow, but expressing directions using head movements is also used as shown in (Gomes et al., 2019). This can replace wheelchairs that are operated by joysticks which is impossible to control for people with disabilities in their upper extremities. Craig and Nguyen (2006) presented the first head motion movement system that uses a telemetric head motion tool to control a powered wheelchair.

Alam et al. (2019; Noman et al., 2018) proposed the development of electrical wheelchair that detects the gesture of hands and finger motion using a mobile 
phone and microcontroller. The microcontroller uses touch sensor and smartphone gesture function to control an electric wheelchair.

In previous works, authors used the automatic speech recognition technique for electric wheelchair control where the most common type of voice control is based on detecting various keywords that function as commands to accomplish a specific motion as reported in (Oleiwi and Alkhalid, 2019; Sinyukov et al., 2014). The voice-controlled method allows the user to use specific commands which can move the wheelchair to different locations rather than saying each command verbally.

Electroencephalogram (EEG) is a method that enables the brain's bioelectric activity to be recorded through electrodes applied to the scalp. This technique could be used as a possible control system coupled with the use of algorithms that classify distinct patterns in the recorded brain activity (Wang et al., 2014). Barriuso et al. (2018) stated that EEG signals can be used to control the movement of an electric wheelchair inside a closed environment with minimal modification to its structure which is also safe and minimally low cost. Jameel et al. (2020) proposed a system to control wheelchair by using human head motion detected by an instrument called EMOTIV Insight.

Abiyev et al. (2016; Al-Aubidy, 2019) presented Wavelet Transform (WT) method to be used for the extraction of the mental tasks. WT coefficients provide the highest discrimination in the appropriate frequency band between wheelchair instructions. The system which binds brain activity to external operations such as multiple microcontrollers that can operate an electric wheelchair is called the Brain Computer Interface (BCI) and involves both invasive and non-invasive methods where there are several primary non-invasive modalities, including EEG and other parameters could be used. There are possible four commands, two generated from the prefrontal cortex and two from the motor cortex by configuring functional Near Infrared Spectroscopy (fNIRS) and EEG to generate each control signal from its associated brain region (Khan et al., 2014; Khan and Hong, 2017).

Various methods of controls are reported, such as breathing pressure that causes the wheelchair to move, another method proposed by (Caiza et el., 2020) is used the tracking eye movement, blink detection and eye gaze tracker where it tracks the eyes causing the wheelchair to move in response to its motion. In such devices there is no need to use a coupling medium to extract signals (Nguyen and Jo, 2012).
Taher et al. (2016; Mohd et al., 2020) have shown that the development of eye detection technology gives the chance to incorporate eyes to be used in assistive devices. This advancement in technology gives the ability of detecting eye signals and eyetracking, so it can be used as an input signal for many application systems as reported in (Çetinel et al., 2017; Ma'touq et al., 2014).

Eye-tracking is accomplished by using a camera to obtain user images and evaluate user intent, where a camera is set up to capture the user's face in real time and then data handling module extracts horizontal and vertical eye positions which can be determined from the pupil major coordinates (Li et al., 2019; Veerati et al., 2018). For safety reasons, an emergency stop is triggered when the electric wheelchair user does not concentrate his eyes consistently in one direction for a specified time.

The rest of this paper is divided as follows: Section II introduces the background, section III describes the methodology, section IV presents the results, section V discusses these results and section VI concludes the findings of the paper.

\section{Background}

Cooper et al. (2006) concluded that a convenient well-designed wheelchair in general has several features that contribute to the rehabilitation of the user such as seat height, seat width, backrest height, seat and back angles, armrests, foot-rests, rear-wheel camber, leg rests, anti-tip devices, tilt and recline.

Various types of microcontrollers are used to control processes, among them is the Arduino, which is an opensource microcontroller that can be programmed to give output voltage in a specific time and magnitude (Components 101, 2020). Power wheelchairs are similarly structured having the following basic parts: Chair with wheels, motor, battery, drive system and controller. The lightweight power wheelchair uses a 2pole motor, while the heavy-duty power wheelchair uses a 4-pole motor. The 4-pole motor offers more carrying power and allows for additional important options.

Since Controlling the wheelchair with a joystick isn't convenient to many individuals with severe motordisabilities such as cerebral palsy, high level spinal cord injury and poliomyelitis, multiple controls can be manipulated such as.

\section{A. Voice Control}

To remove the need to move one or more limbs to command the seat, (Malik et al., 2017) proposed a 
voice controlled system to help the wheelchair user to maintain a convenient location within his seat frame. It is more compatible yet more convenient to talk rather moving the user limbs to control the wheelchair according to the results obtained by (Bramhe et al., 2017; Agarwal et al., 2019).

\section{B. Head Motion Control}

Machangpa and Chingtham (2018) reported that the head development is one of the most straightforward and normally followed signals to control a wheelchair. Other researchers (Prasad et al., 2017; Ankit et al., 2018) concluded that the wheelchair control systems with the head requires high concentration and precise movement of the head which could cause neck pain in rare cases.

\section{Sip and Puff Control}

The Sip-and-puff controller is an assistive technology that sends signals using air pressure by Sipping or puffing through a tube in order to move the electric wheelchair which is perfect for individuals who have restricted limbs movements to operate the wheelchair and for people affected with severe motor disabilities, as manipulating a standard switch can be difficult, tiring and in some cases impossible (Riman, 2018).

\section{Finger Control}

Finger switch is a type of electric wheelchair control which could enable people who suffer from a disability to move freely from one place to another by using their fingers as a joystick where the user has to bend his fingers to move a wheelchair (Megalingam et al., 2017).

\section{E. Eye Control}

Electromyogram is the signal produced by the movement of the eye and can be used to control mobility aids. EOG is captured by placing 5 electrodes around the eyes. Two electrodes are placed to the right and left of the outer canthi and two electrodes are placed to detect horizontal movements and one reference electrode. Eye movement could be detected by using various methods as reported in (Abraham et al., 2019; Jafar et al., 2019).

In this study, a novel approach to wheelchair design, control and operation is proposed and demonstrated. The new approach uniquely combines and correlates five physiological variables to control the wheelchair activities namely; voice, breathing pressure, head-movements, finger gestures and eye movements. These variables are selected because they are the least damaged parameters after having an accident that could hinder the mobility of the body. These variables are most critical and sensitive for a balanced control of the wheelchair motion and also conserve much needed energy compared with pushing the rims manually. The built wheelchair proved to be inexpensive with ability to apply various controlling algorithms to improve both the comfortability and the safety of the patient.

\section{Methodology}

The system is designed and tested using the flowchart in Fig. 1 as follows.

When the push button is not pressed, the counter value is 0 , which indicates that the voice control is activated. If the push button is pressed once, the counter value is one, which indicates that breathing pressure control is activated. If the push button is pressed twice, the counter value becomes two, which indicates that head movement control is activated. If the push button is pressed a third time, the counter value becomes three, which indicates that the finger flex control is activated. If the push button is pressed a fourth time, the counter value becomes four, which indicates that the EOG control is activated. Finally, if the push button is pressed a fifth time, the counter value resets back to 0 , which means that voice control is activated.

The final operational system, proposed by the author comprises the modules as presented in Fig. 2.

\section{A. Voice Control System}

The voice control system is composed from a microcontroller, Voice Recognition Module (VRM) and H-bridge. This control begins when the patient provides a voice command to the VRM which is mainly used to match the voice commands given by the user and the nature of voice command recorded in the VRM, then the signal is conveyed from the VRM to the microcontroller, which interprets the electrical signals into mechanical motion of the motors through the H-bridge, resulting in the wheelchair movement.

\section{B. Head Movement System}

The head movement system is designed using accelerometer (MPU-6050), transceiver (nRF24L01), microcontroller and H-bridge. When the patient tilts his head in a particular degree and direction, the accelerometer senses the direction of tilting and translates it into wheelchair movement through the microcontroller and the $\mathrm{H}$-bridge. 


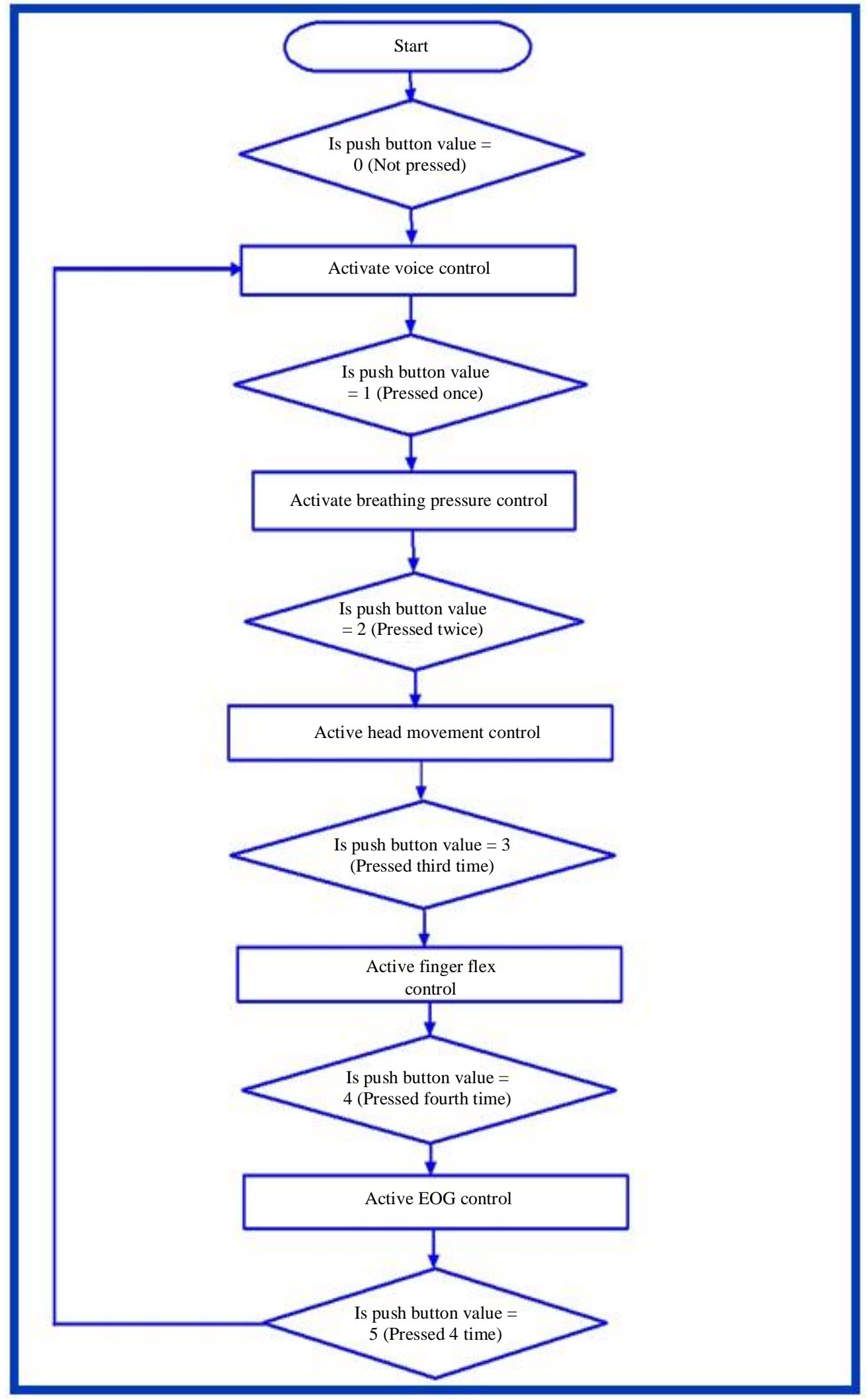

Fig. 1: System's flowchart 


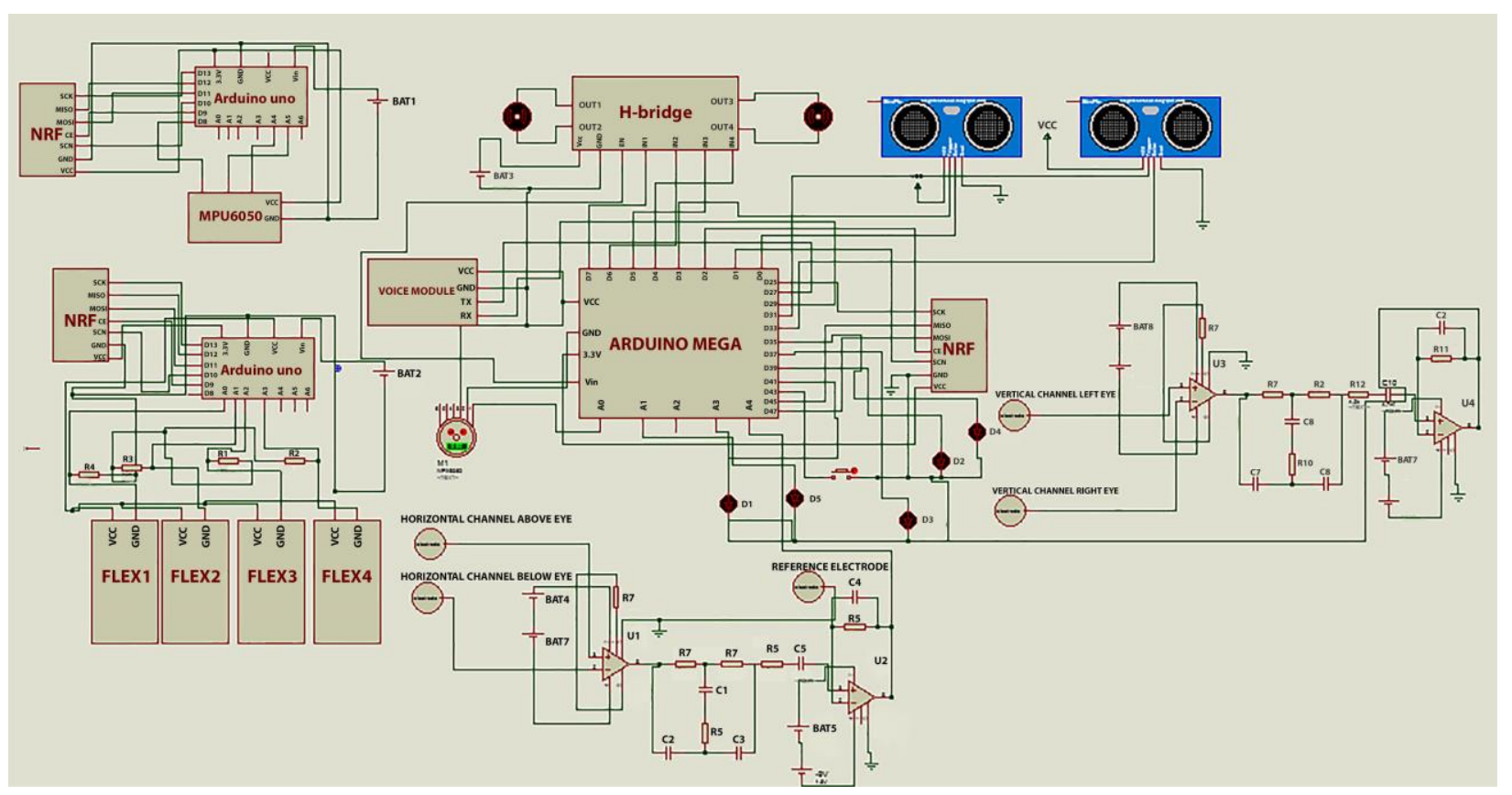

Fig. 2: Overall integrated system to control a wheelchair

\section{Breathing Pressure System}

The breathing pressure system consists of pressure sensor (MPX50GP), pressure tube, microcontroller and H-bridge. The initiation of this module starts when a patient applies breathing pressure into a tube that is connected to the pressure sensor which is then translated to electrical signal to control wheelchair movement through the microcontroller and the H-bridge.

\section{Finger Flexing System}

The finger flex system is constructed from flex sensor, transceiver (nRF24L01), microcontroller and H-bridge, where four fingers indicate different wheelchair directions with a finger flex sensor on each one. When flexing a finger the flex senor converts this mechanical signal into an electrical signal which will be translated into wheelchair movement through the microcontroller and the H-bridge.

\section{E. Eye Control System}

The eye control system is developed using an instrumentation amplifier, a microcontroller, a motor driver and a low pass filter. Where different eye movements indicate different wheelchair movements. When blinking or moving the eye in any direction, the circuit converts the mechanical signal to electrical signal capable to control the wheelchair movement through the microcontroller.

\section{Results}

Figure 3 shows the results of the signal peak values testing of the EOG for the designed wheelchair system, with detailed measurements presented in Tables 1 to 4 .

Figure 4 shows the response time against velocity graph, while Fig. 5 shows the response for eye movement control. Figure 6 shows the wheel controls, whereas Fig. 7 shows wheel chair system prototype, with Fig. 8 to 10 presenting the carried out reliability tests under the control circuit in Fig. 11 which includes.

\section{Voice Control}

The VRM recognizes the voice command which is then processed by the main microcontroller. The motor drivers operate depending on the voice confirmed by the microcontroller and then the motors function depending on the sent voice command.

\section{Head Movement Control}

The accelerometer reads the change in the orientation of the head on the $x-y$ axes, then this data is transmitted into a receiver module and delivered to the main microcontroller, which commands the motors to operate depending on the tilting of the head.

\section{Breathing Pressure Control}

The pressure sensor receives the data acquired by breathing pressure which is then processed by the main microcontroller. Then motor driver operates depending on the pressure volume confirmed by the main microcontroller. Finally the motors function depending on how much pressure is applied. 
Table 1: voice control. Results of the

\begin{tabular}{lll}
\hline Command Voice control & Result & Time required \\
\hline Go & Forward. & $0.8 \mathrm{sec}$ \\
Right & Right. & $1.3 \mathrm{sec}$ \\
Left & Left. & $1.2 \mathrm{sec}$ \\
Break & Stop. & $0.6 \mathrm{sec}$ \\
Back & Backward. & $1.5 \mathrm{sec}$ \\
\hline
\end{tabular}

Table 2: Results of the head control.

\begin{tabular}{lll}
\hline Command Head movement control ( $\mathrm{X}$ and $\mathrm{Y}$ orientation) & Result & Time required \\
\hline $\mathrm{X}=\mathrm{N} / \mathrm{R}, \mathrm{Y}=\mathrm{Y}<135$ & Forward. & $1 \mathrm{sec}$ \\
$\mathrm{X}=\mathrm{N} / \mathrm{R}, \mathrm{Y}=\mathrm{Y}>150$ & Backward. & $1.2 \mathrm{sec}$ \\
$\mathrm{X}=\mathrm{X}>365, \mathrm{Y}=\mathrm{N} / \mathrm{R}$ & Left. & $1.3 \mathrm{sec}$ \\
$\mathrm{X}=\mathrm{X}<345, \mathrm{Y}=\mathrm{N} / \mathrm{R}$ & Right. & $1.3 \mathrm{sec}$ \\
$\mathrm{X}=360>\mathrm{X}>345, \mathrm{Y}=150>\mathrm{y}>140$ & Stop & $1.5 \mathrm{sec}$ \\
\hline
\end{tabular}

Table 3: Results of the pressure control.

\begin{tabular}{lll}
\hline Command Pressure control (Kpa) & Result & Time required \\
\hline $35>=$ Pressure value $>=20$ & Forward. & $0.9 \mathrm{sec}$ \\
$3>=$ Pressure value $>=2$ & Left. & $1.2 \mathrm{sec}$ \\
$17>=$ Pressure value $>=13$ & Right. & $0.8 \mathrm{sec}$ \\
$9>=$ Pressure value $>=8$ & Stop. & $1.1 \mathrm{sec}$ \\
\hline
\end{tabular}

Table 4: Test results of the finger flexing control

\begin{tabular}{lll}
\hline Command Finger flexing control (Flexing Range) & Result & Time required \\
\hline Flexing of thumb finger (20 to 30) & Backward. & $1.7 \mathrm{sec}$ \\
Flexing of index finger (30 to 50) & Left. & $1.3 \mathrm{sec}$ \\
Flexing of middle finger (40 to 50) & Forward. & $1.5 \mathrm{sec}$ \\
Flexing of ring finger (40 to 50) & Right. & $1.1 \mathrm{sec}$ \\
None (51 to 80) & Stop. & $0.8 \mathrm{sec}$ \\
\hline
\end{tabular}

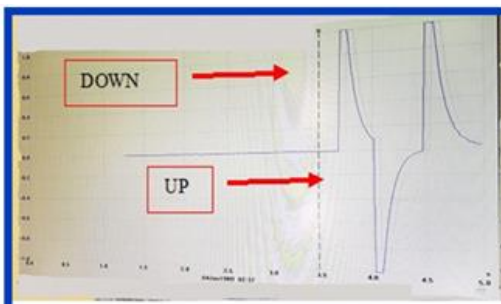

(a) Movement of the eyeball up and down

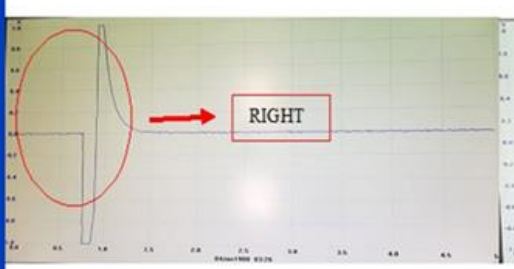

(c) Eyeball movement to the right

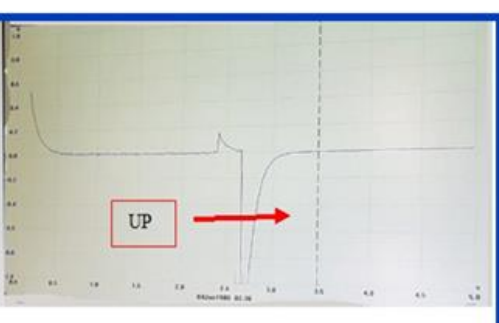

(b) Eyeball movement up

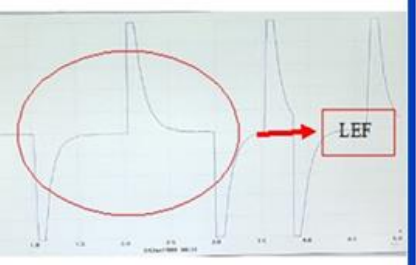

(d) Eyeball movement to the left

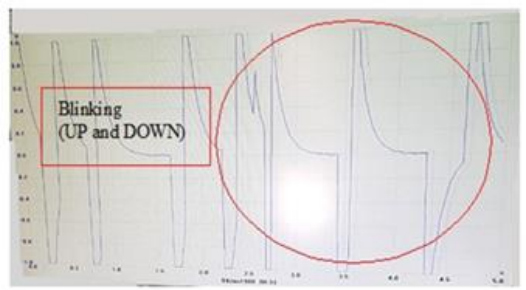

(e) Blinking movement

Fig. 3: Eye movements tracking signals 


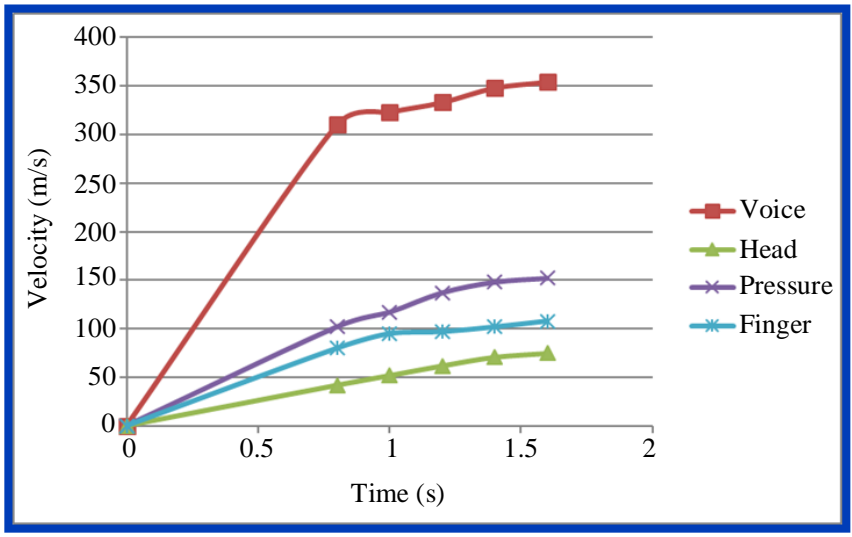

Fig. 4: Response time against velocity graph

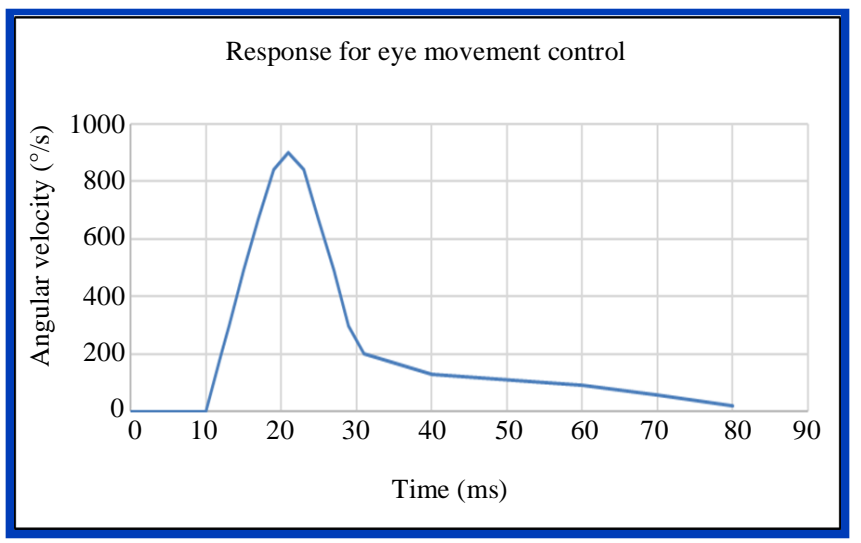

Fig. 5: Response time against angular velocity graph of eye movement control

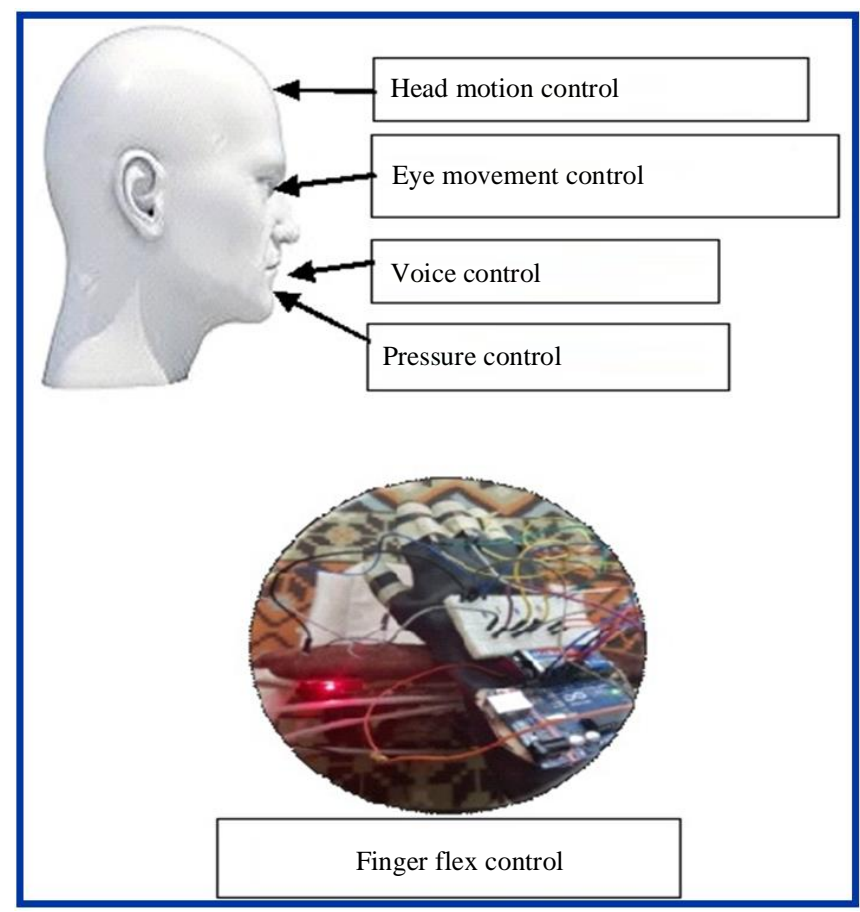

Fig. 6: System controls 


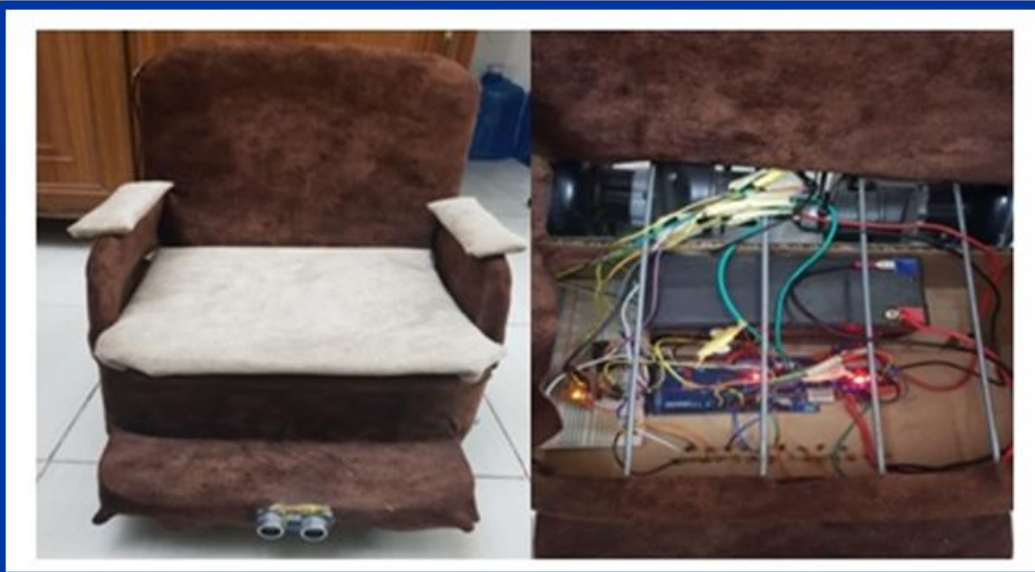

Fig. 7: Wheelchair prototype

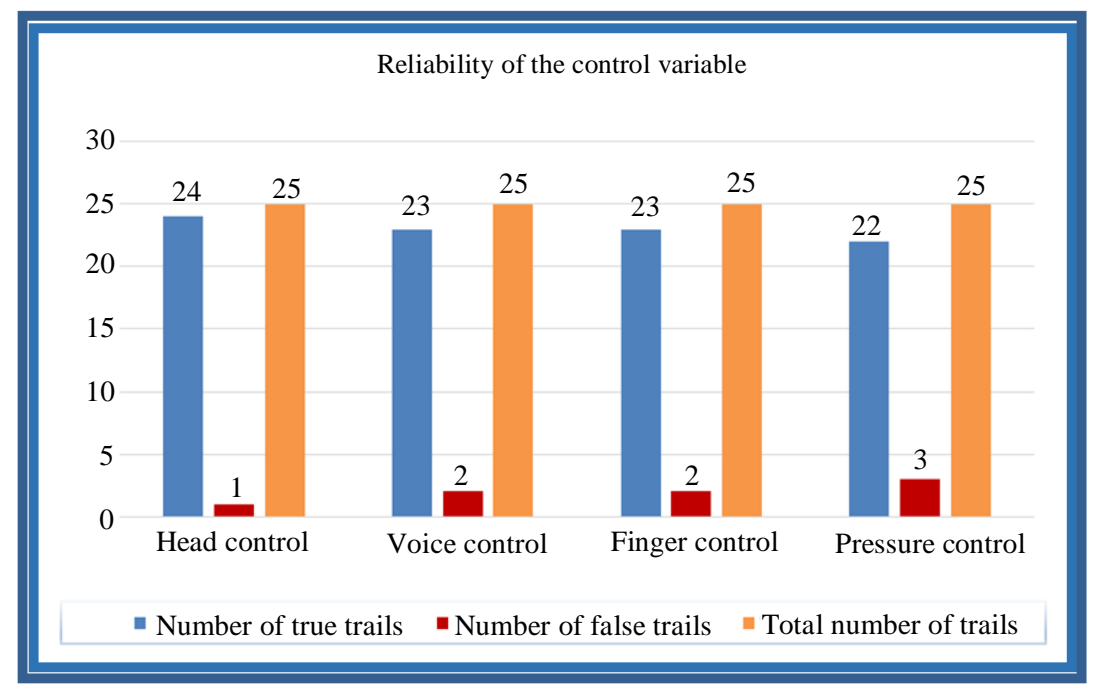

Fig. 8: System reliability test

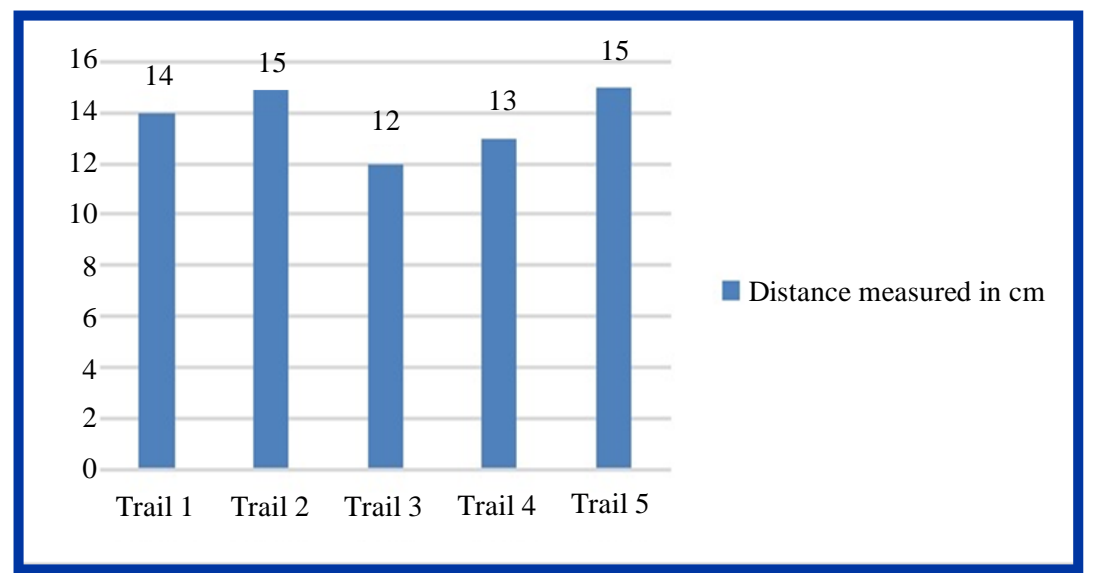

Fig. 9: System safety with ultrasound sensor at 90 degrees 


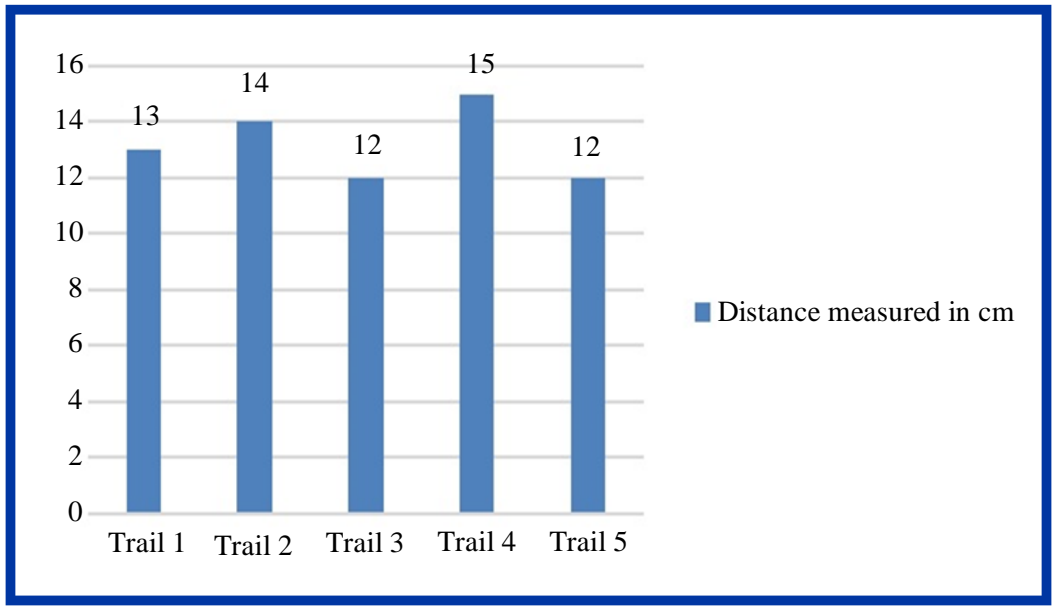

Fig. 10: System safety with ultrasound sensor at 45 degrees

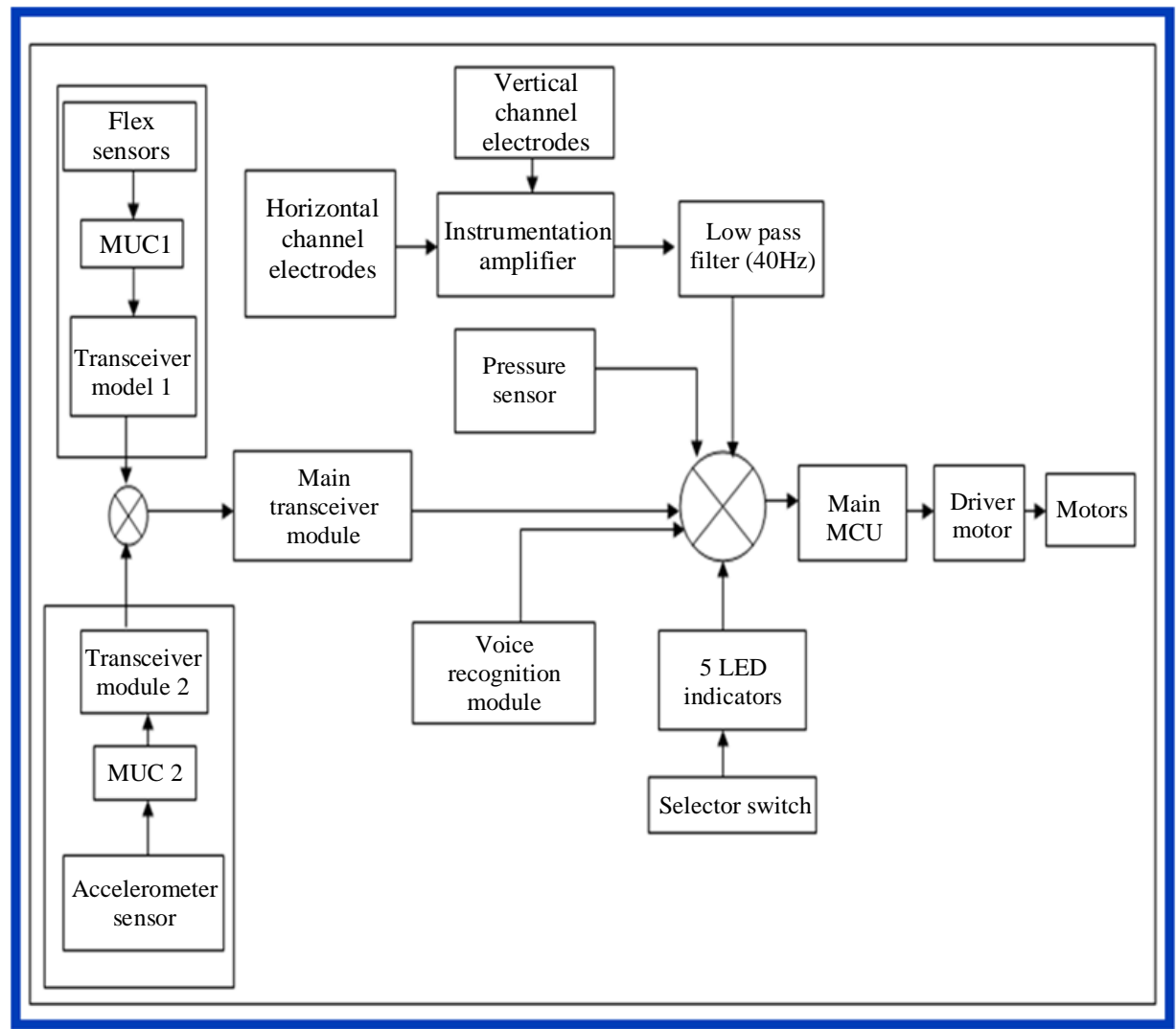

Fig. 11: System control block

\section{Finger Flex Control}

Bending the fingers in certain degrees gives different electrical resisting values acquired by the flex sensor, the data is then transmitted from transceiver module to the main microcontroller where the data is processed. Then motor driver operates the motors and the wheelchair is moved accordingly.

\section{EOG Control}

The electrical signal is acquired using electrodes placed on the eyes that are amplified using an instrumentation amplifier. The amplified signal is then filtered using low pass filter with a frequency of $40 \mathrm{~Hz}$. The filtered signal is then processed in the main microcontroller, depending on the signal, the motor 
driver operates and the motors function depending on the eye signal extracted.

The ultrasound sensor is used as the safety aspect where it cuts off the movement of the wheelchair when an obstacle or stairs come across the wheelchair path.

\section{Discussion}

The designed and developed wheelchair prototype with various interfacing modules was accomplished in this study, the movement of wheelchair was tested using different physiological variables. The results showed that the wheelchair moved according to the voice command; forward when the user said (go), to the right when the user said (right), to the left when the user said (left) and stopped when the user said (break) as shown in Table 1.

The head movement control system was designed and tested. The wheelchair moved depending on the angular orientation of the head as follows; moved forward when the user tilted his head forward only for a maximum of 8 sec for the safety of the user because if the patient suffers a stroke, his head may tilt forward which will result in the wheelchair keeping to move forward, hence, avoiding neck injury. The wheelchair moved right when the user tilted his head right for a maximum of $5 \mathrm{sec}$, moved left when the user tilted his head left for a maximum of $5 \mathrm{sec}$ and stopped when the user centered his head in a certain $\mathrm{x}-\mathrm{y}$ point. The readings of the sensor used to detect the head movements are converted to digital data and entered the microcontroller which translates this data to orders to control the motors of the wheelchair. The system programmed and calibrated in a manner that right and left movements depend on the change in the chosen $\mathrm{X}$ values, whereas forward and backward movements depend on the chosen $\mathrm{Y}$ values. The calibrated values and results are presented in Table 2. As example if $X$ value less than 150, the microcontroller gives order to the motors to move the wheelchair forward.

The wheelchair functionality is further improved through the detection of shaking of the head, eye, fingers and abnormal breathing which can be achieved by using Bessel low pass filter that allows the normal action movement of the head, eye, fingers and normal breathing which will cause the system not to respond to abnormal action of shaking source of control and the wheelchair will stop.

The breathing control circuit accomplished what it was intended for. When a high breathing pressure is applied, the wheelchair moves forward, when a lower breathing pressure is applied, the wheelchair moves to the right, when the user applies a pressure into his lungs the wheelchair moves to the left and when the user stops breathing into the tube, the wheelchair stops moving. The pressure sensor used in this design detects the change in pressure during snipping and puffing of the disabled individual in the tube and converts this change to digital data to be entered to the microcontroller and then to be translated into a certain values according to the programming of the microcontroller. These values and thresholds determine the direction of the wheelchair movement. These control values and the results of testing breath control circuit are shown in Table 3. From this table, it's clear that if the pressure value between 20 35 , the microcontroller gives an appropriate signal to the motors of the wheelchair, via H-bridge, to move forward.

The finger flexing control system functioned properly. The test results of this system are shown in Table 4. The wheelchair moved depending on the flexing of the fingers of the impaired individual. When the impaired flexes his thumb the wheelchair moves backwards for a maximum of $5 \mathrm{sec}$ for the safety of patient because if the user gets a seizure, the wheelchair doesn't keep moving. When the index finger is flexed, it moves left for a maximum $5 \mathrm{sec}$, when the middle finger is flexed it moves forward for a maximum $8 \mathrm{sec}$ and finally when the patient flexes his ring finger the wheelchair moves right for a maximum $5 \mathrm{sec}$.

The EOG control operated as required, the wheelchair moved depending on the eye movement of the patient. When the patient moves his eyes UP the wheelchair moves forward, if the patient moves his eyes RIGHT the wheelchair moves right, if the patient moves his eyes LEFT the wheelchair moves left and finally, if the patient moves his eyes DOWN the wheelchair moves backward, as shown in Fig. 3.

Figure 4 shows that the velocity of voice is the fastest with an average of $334 \mathrm{~m} / \mathrm{s}$. The air pressure flow velocity comes second with an average velocity of $131 \mathrm{~m} / \mathrm{s}$. The finger flexing average velocity is 97 $\mathrm{m} / \mathrm{s}$. The head movement average velocity is $60 \mathrm{~m} / \mathrm{s}$. The minimum response time was $0.8 \mathrm{sec}$ and the maximum is $1.7 \mathrm{sec}$.

The graph in Fig. 5, shows the measured angular velocity of the eye in $(\%)$. The angular velocity of the eye is between $750 \%$ s and $900 \%$

Comparing with the manual wheelchair the proposed electric wheelchair performs the same functionality more efficiently. This wheelchair serves impaired individuals with various spinal cord injuries using five different controls as shown in Fig. 6. The proposed system's prototype is shown in Fig. 7.

Testing the system's reliability was one of the main objectives, so the system is validated through intensive experimental work. Each control method is put into a trail of 25 times with a 5 min waiting interval between each experiment and the correct response per experiment is recorded. The results are shown in Fig. 8. The accuracy of the system is calculated from the obtained results in Fig. 8. It is found to be $88 \%$ for pressure 
control module, $92 \%$ for both finger control and voice control and $96 \%$ for head movement control.

The safety system in the wheelchair consisted of two ultrasound sensors; one is positioned 90 degrees from the ground on the front side of the wheelchair (position 1) and the second is positioned 45 degrees from the ground, so it can stop the wheelchair from moving when it is close to stairs (position 2). The experiment (trail) was repeated five times for each position and the wheelchair stopped at distance $(12-15) \mathrm{cm}$. away from the obstacle in all experiments, for both positions. The results of these experiments are depicted in Fig. 9 and 10.

\section{Conclusion}

In conclusion, a major challenge is to help physically disabled people, increase their mobility and rely on themselves. This challenge is increased when even the upper limbs are disabled. In this case, an ordinary joystick controlled electric wheelchair is not suitable to provide autonomous user movements. The proposed powered wheelchair was developed to help individuals with different spinal cord injuries and illnesses of poliomyelitis that are widespread worldwide. A novel approach to wheelchair design, control and operation is proposed and demonstrated. The new approach uniquely combine and correlate five physiological variables to control the wheelchair activities namely; voice, breathing pressure, head-movements, finger gestures and eye movements. These variables are selected because they are the least damaged parameters after having an accident that could hinder the mobility of the body. The proposed wheelchair is a multicontrol device with ability to choice the optimum method of control according to health condition of the disabled individual easily and smoothly. The designed wheelchair is cost effective which makes it affordable for the community and the user.

The future direction of the proposed work could be concentrated in adding other methods of wheelchair's control by using various physiological variables such as Electromyogram (EMG) and EEG controls, where brain activity could be used to control the electric wheelchair by means of multiple microcontrollers known as BCI. Also it will be valuable to implement WT to extract different features of EEG signal, especially the mental ones that rise upon thinking about certain action to accomplish namely thinking about the wheelchair movement.

Also, an automatic selection control mode of the wheelchair, according to the user needs, could be added in the future work.

The five chosen controls in this study should cover all possible cases of disability. Furthermore, the wheelchair is simple, user-friendly. Depending on the degree of injury, the chosen variables can be calibrated so it responds accordingly.

\section{Acknowledgement}

This work is supported and sponsored by Al-Ahliyya Amman University. The experimental work was carried out by: Talal Altabba, Mahmoud Qamhieh, Mostafa Abuthuaba and Momen Alkhawandi.

\section{Funding Information}

The experimental work and publication is partially funded by Al-Ahliyya Amman University.

\section{Ethics}

There is no conflicts of interest regarding the publication "A novel approach to wheelchair design and operation using multi-function controller".

\section{References}

Abiyev, R. H., Akkaya, N., Aytac, E., Günsel, I. and Çağman, A. (2016). Brain-computer interface for control of wheelchair using fuzzy neural networks. BioMed research international, 2016.

Abraham, R., Thaliath, R. M., Davies, S., Jacob, T. and Johny, T. (2019). Eye Controlled Wheelchair with Asthenopia Detection. In 2019 3rd International conference on Electronics, Communication and Aerospace Technology (ICECA) (pp. 502-505). IEEE.

Agarwal, T., Bhalgat, S., Khandhar, C. and Khetan, B. (2019). Swheel: Low-Cost Smart Wheelchair with Wireless Control. In 2019 International Conference on Communication and Electronics Systems (ICCES) (pp. 1021-1025). IEEE.

Alam, M. E., Kader, M. A., Hany, U., Arjuman, R., Siddika, A. and Islam, M. Z. (2019). A multicontrolled semi-autonomous wheelchair for old and physically challenged people. In 2019 1st International Conference on Advances in Science, Engineering and Robotics Technology (ICASERT) (pp. 1-6). IEEE.

Al-Aubidy, K. M. (2019). Wheelchair Neuro Fuzzy Control Using Brain Computer Interface. In 2019 12th International Conference on Developments in eSystems Engineering (DeSE) (pp. 640-645). IEEE.

Ankit, K., Vikas, C. Kuldeep, S. Avinash Raj, Y. Ishwar, Y. Pankaj D. and Somkuwar. S. (2018). HeadMotion Controlled Wheel Chair Direction using ATMega328p Microcontroller. International Journal of Advanced Research in Computer and Communication Engineering, 7 (4), 61-65.

Barriuso, A. L., Pérez-Marcos, J., Jiménez-Bravo, D. M., Villarrubia Gonzalez, G. and De Paz, J. F. (2018). Agent-based intelligent interface for wheelchair movement control. Sensors, 18(5), 1511. 
Bramhe, M. V., Vijay, N., Rao, K. B., Bisen, P., Navsalkar, R. and Bajganiya, T. (2017). Voice Controlled Wheelchair for Physically Disabled Person. International Journal of Advanced Research in Electrical, Electronics and Instrumentation Engineering, 6(2), 940-948.

Caiza, G., Reinoso, C., Vallejo, H., Albarracín, M. and Salazar, E. P. (2020). Semi-automatic Eye Movement-Controlled Wheelchair Using Low-Cost Embedded System. In World Conference on Information Systems and Technologies (pp. 755764). Springer, Cham.

Çetinel, G., Sevda, G. Ü. L., Tiryaki, Z., Enes, K. U. Z. U. and Milligüney, M. (2017). Move Your Wheelchair with Your Eyes. International Journal of Applied Mathematics Electronics and Computers, (Special Issue-1), 5-8.

Chow, J. W. and Levy, C. E. (2011). Wheelchair propulsion biomechanics and wheelers' quality of life: An exploratory review. Disability and Rehabilitation: Assistive Technology, 6(5), 365-377.

Components 101, (2020) a resource dedicated for electronics design engineers 2018, Arduino Uno schematic

diagram..https://components101.com/microcontrolle rs/arduino-uno

Cooper, R. A., Ohnabe, H. and Hobson, D. A. (Eds.). (2006). An introduction to rehabilitation engineering. CRC Press.

Craig, D. A. and Nguyen, H. T. (2006). Wireless realtime head movement system using a Personal Digital Assistant (PDA) for control of a power wheelchair. In 2005 IEEE Engineering in Medicine and Biology 27th Annual Conference (pp. 772-775). IEEE.

Ebrahimi, A., Kazemi, A. and Ebrahimi, A. (2016). Wheelchair Design and Its Influence on Physical Activity and Quality of Life Among Disabled Individuals. Iranian Rehabilitation Journal, 14(2), 85-92.

Gomes, D., Fernandes, F., Castro, E. and Pires, G. (2019). Head-movement interface for wheelchair driving based on inertial sensors. In 2019 IEEE 6th Portuguese Meeting on Bioengineering (ENBENG) (pp. 1-4). IEEE.

Jafar, F., Fatima, S. F., Mushtaq, H. R., Khan, S., Rasheed, A. and Sadaf, M. (2019). Eye Controlled Wheelchair Using Transfer Learning. In 2019 International Symposium on Recent Advances in Electrical Engineering (RAEE) (Vol. 4, pp. 1-5). IEEE.

Jameel, H. F., Mohammed, S. L. and Gharghan, S. K. (2020). Wheelchair Control System based on Gyroscope of Wearable Tool for the Disabled. MS\&E, 745(1), 012091.
Khan, M. J. and Hong, K. S. (2017). Hybrid EEGfNIRS-based eight-command decoding for BCI: Application to quadcopter control. Frontiers in neurorobotics, 11, 6 .

Khan, M. J., Hong, M. J. and Hong, K. S. (2014). Decoding of four movement directions using hybrid NIRS-EEG brain-computer interface. Frontiers in human neuroscience, 8, 244.

Li, Y., Huang, Q., Zhang, Z., Yu, T. and He, S. (2019). An EEG-/EOG-Based Hybrid Brain-Computer Interface: Application on Controlling an Integrated Wheelchair Robotic Arm System. Frontiers in Neuroscience, 13, 1243.

Ma'touq, J., Al-Nabulsi, J., Al-Kazwini, A., Baniyassien, A., Al-Haj Issa, G. and Mohammad, H. (2014). Eye blinking-based method for detecting driver drowsiness. Journal of Medical Engineering \& Technology, 38(8), 416-419.

Machangpa, J. W. and Chingtham, T. S. (2018). Head Gesture Controlled Wheelchair for Quadriplegic Patients. Procedia computer science, 132, 342-351.

Malik, M. I., Bashir, T. and Khan, O. F. (2017). Voice controlled wheel chair system. International Journal of Computer Science and Mobile Computing, 6(6), 411-419.

Mann, W. C., Goodall, S., Justiss, M. D. and Tomita, M. (2002). Dissatisfaction and nonuse of assistive devices among frail elders. Assistive Technology, 14(2), 130-139.

Megalingam, R. K., Sreekanth, S., Govardhan, A., Teja, C. R. and Raj, A. (2017). Wireless gesture controlled wheelchair. In 2017 4th International Conference on Advanced Computing and Communication Systems (ICACCS) (pp. 1-5). IEEE.

Mohd, A. A. Q., Khairudin, M. F. M., Basirun, L. H. and Jailani, R. (2020). Motorised Wheelchair with Multicontrol System. In 2020 IEEE 10th Symposium on Computer Applications \& Industrial Electronics (ISCAIE) (pp. 233-238). IEEE.

Nguyen, Q. X. and Jo, S. (2012). Electric wheelchair control using head pose free eye-gaze tracker. Electronics Letters, 48(13), 750-752.

Noman, A. T., Khan, M. S., Islam, M. E. and Rashid, H. (2018). A New Design Approach for Gesture Controlled Smart Wheelchair Utilizing Microcontroller. In 2018 International Conference on Innovations in Science, Engineering and Technology (ICISET) (pp. 64-68). IEEE.

Oleiwi, B. K. and Alkhalid, F. F. (2019). Smart Autonomous Wheelchair Controlled by Voice Commands-Aided by Tracking System. Iraqi Journal OF Computers, Communication and Control and Systems Engineering, 19(1), 82-87.

Philip, E. (2012). Eubserver, 2012. Disability in Figures. https://euobserver.com/disability/118249 
Prasad, S., Sakpal, D., Rakhe, P. and Rawool, S. (2017). Head-motion controlled wheelchair. In 2017 2nd IEEE International Conference on Recent Trends in Electronics, Information \& Communication Technology (RTEICT) (pp. 1636-1640). IEEE.

Riman, C. F. (2018). Multi-Controlled Wheelchair for Upper Extremities Disability.

Sinyukov, D. A., Li, R., Otero, N. W., Gao, R. and Padir, T. (2014). Augmenting a voice and facial expression control of a robotic wheelchair with assistive navigation. In 2014 IEEE International Conference on Systems, Man and Cybernetics (SMC) (pp. 10881094). IEEE.

Taher, F. B., Amor, N. B. and Jallouli, M. (2016). A self configured and hybrid fusion approach for an electric wheelchair control. In 2016 IEEE 8th International Conference on Intelligent Systems (IS) (pp. 729-734). IEEE.
Taylor, D. M. (2018). Americans with disabilities: 2014. US Census Bureau.

Thompson, S. (2018). The current situation of persons with disabilities in Jordan.

Veerati, R., Suresh, E., Chakilam, A. and Ravula, S. P. (2018). Eye Monitoring Based Motion Controlled Wheelchair for Quadriplegics. In Microelectronics, Electromagnetics and Telecommunications (pp. 4149). Springer, Singapore.

Wang, H., Li, Y., Long, J., Yu, T. and Gu, Z. (2014). An asynchronous wheelchair control by hybrid EEGEOG brain-computer interface. Cognitive neurodynamics, 8(5), 399-409. 